\title{
Dihydroartemisinin triggers ferroptosis in primary liver cancer cells by promoting and unfolded protein response-induced upregulation of $\mathrm{CHAC1}$ expression
}

\author{
ZHIWEI WANG ${ }^{1,2^{*}}$, MINGXING LI ${ }^{1,2}$, YUANFENG LIU ${ }^{1}$, \\ ZHENTAO QIAO $^{1,2}$, TAO BAI ${ }^{1,2^{*}}$, LING YANG ${ }^{2}$ and BO LIU ${ }^{2}$
}

Departments of ${ }^{1}$ Vascular and Endovascular Surgery, and ${ }^{2}$ Hepatopancreatobiliary Surgery, The First Affiliated Hospital of Zhengzhou University, Zhengzhou, Henan 450052, P.R. China

Received March 15, 2021; Accepted August 16, 2021

DOI: $10.3892 /$ or.2021.8191

\begin{abstract}
Dihydroartemisinin (DHA), an artemisinin derivate, has been investigated as a potential antitumor drug in primary liver cancer (PLC). Ferroptosis is a form of iron-dependent cell death that can be driven by lipid peroxidation inducers. The present study aimed to determine whether and how DHA could promote the death of PLC cells by inducing ferroptosis. In total, four PLC cell lines with different p53 statuses, including Hep3B (p53 null), Huh7 (p53 mutant), PLC/PRF/5 (p53 mutant) and HepG2 (p53 wild-type), were treated with various concentrations of DHA. The effects of DHA on all three branches of the unfolded protein response (UPR) were evaluated. To deactivate the UPRs, small interfering RNA was used to knockdown the expression of activating transcription factor (ATF) 4, X-box binding protein 1 (XBP1) or ATF6 in PLC cells. The effect of DHA on the promoter activity of Chac glutathione specific $\gamma$-glutamylcyclotransferase 1 (CHACl) was evaluated using a dual luciferase reporter assay. The results revealed that DHA-induced death in PLC cells was irrelevant of the p53 status. PLC cells exposed to DHA displayed classic features
\end{abstract}

Correspondence to: Dr Tao Bai, Department of Vascular and Endovascular Surgery, The First Affiliated Hospital of Zhengzhou University, 1 East Jianshe Road, Zhengzhou, Henan 450052, P.R. China E-mail: doctorbaitao@163.com

\section{${ }^{*}$ Contributed equally}

Abbreviations: DHA, dihydroartemisinin; ROS, reactive oxygen species; MDA, malondialdehyde; GSH, glutathione; GSSG, oxidized glutathione; GPX4, glutathione peroxidase 4; PERK, protein kinase R-like ER kinase; eIF2, eukaryotic initiation factor 2; ATF4, activating transcription factor 4; ATF6, activating transcription factor 6; IRE1 $\alpha$, inositol-requiring transmembrane kinase/endoribonuclease $1 \alpha$; $\mathrm{XBP} 1, \mathrm{X}$ box-binding protein 1; CHAC1, ChaC glutathione specific $\gamma$-glutamylcyclotransferase 1; SLC7A11, solute carrier family 7 member 11; SLC3A2, SLC family 3 member 2

Key words: DHA, ferroptosis, unfolded protein response, $\mathrm{CHAC1}$ of ferroptosis, such as increased lipid reactive oxygen species and malondialdehyde levels, an iron overload, and decreased activity or expression of glutathione (GSH), glutathione peroxidase 4, solute carrier family (SLC) 7 member 11 and SLC family 3 member 2 . The antitumor effects of DHA in PLC cells were significantly weakened by two typical ferroptosis inhibitors, ferrostatin-1 and deferoxamine mesylate salt, whereas the antitumor effects were augmented following iron overload. Furthermore, DHA activated all three branches of the UPR (eukaryotic translation initiation factor $2 \alpha$ kinase 3/eukaryotic translation initiation factor $2 \mathrm{~A} / \mathrm{ATF} 4$, inositol-requiring transmembrane kinase/endoribonuclease $1 \alpha / \mathrm{XBP} 1$ and ATF6 branches) in vitro. Notably, DHA-induced ferroptosis was significantly attenuated following the knockdown of ATF4, XBP1 or ATF6 expression. In addition, the promoter activity of $\mathrm{CHAC1}$, a gene capable of degrading GSH, was enhanced by DHA, but weakened when the aforementioned three UPR transcription factors were knocked down. In conclusion, the findings of the present study suggested that DHA may effectively induce ferroptosis in PLC cells through the activation of anti-survival UPRs and the upregulation of CHAC1 expression.

\section{Introduction}

Primary liver cancers (PLCs), of which the most predominant type is hepatocellular carcinoma, are the third leading cause of cancer-related mortality worldwide after lung cancer $(1,2)$. The morbidity of PLCs markedly varies between geographic regions around the world, with the highest rates observed in sub-Saharan Africa and Eastern Asia (3). The major risk factors for PLC include chronic hepatitis $\mathrm{B} / \mathrm{C}$ virus infection, excessive alcohol consumption, non-alcoholic fatty liver disease and aflatoxin exposure $(3,4)$. Although current treatments, including percutaneous local ablation, surgical resection and liver transplantation, improve the survival of patients with PLC, the mortality rate continues to increase due to population growth and high recurrence rates $(1,5,6)$. Therefore, further molecular studies on PLCs are urgently required to identify novel targets for PLC treatment.

Ferroptosis is an iron-dependent form of non-apoptotic cell death first reported by Dixon et al (7) in 2012. This type of cell death is caused by lethal lipid peroxidation and its typical 
features, such as the overproduction of lipid reactive oxygen species (ROS) and the impairment of crista and outer mitochondrial membrane (8), are different from other types of regulated cell death. Solute carrier family 7 member 11 (SLC7A11) and solute carrier family 3 member 2 (SLC3A2) control the import of extracellular cysteine and cystine, and regulate glutathione (GSH) synthesis (9). GSH depletion leads to the suppression of glutathione peroxidase (GPX)4, a key glutathione peroxidase known to catalyze the reduction of lipid ROS (9).

One of the major reasons for therapeutic resistance in cancer was discovered to be the intrinsic or acquired resistance of cancer cells to executioner-mediated apoptosis (10). Compared with non-cancer cells, cancer cells often required increased iron to promote their proliferation. Interestingly, this iron dependency also made cancer cells more sensitive to ferroptosis (11). Therefore, small molecules, such as erastin, sorafenib and Ras-selective lethal small molecule-3/-5, which were found to be capable of inducing iron-dependent accumulation of lipid ROS, are currently being evaluated for their potential in cancer therapy $(12,13)$. The identification of drugs able to induce ferroptosis may provide novel insights into the potential of ferroptosis as a novel target for PLC treatment.

Artemisinin is a sesquiterpene trioxane lactone originally extracted from Artemisia annua L., and its derivates have been discovered to be effective anti-malarial agents (14). To date, in addition to its well-known anti-malarial application, artemisinins are currently being evaluated for their potential in treating multiple cancer types due to their established safety recorded in thousands of patients with malaria (14). Ooko et al (15) treated 60 cancer cell lines with 11 artemisinin derivates, and analyzed the expression profiles of 30 iron-related genes in these cell lines via microarray hybridization. The results found that the $\mathrm{IC}_{50}$ values of analyzed artemisinins were significantly correlated with the expression of $\geq 20$ iron-related genes. These findings suggested the involvement of artemisinins in the induction of ferroptosis in cancer cells. Artesunate is the most frequently reported derivate of artemisinin, which has been found to trigger ferroptosis in malignant cells $(16,17)$. Dihydroartemisinin (DHA), a semi-synthetic derivative of artemisinin, has been demonstrated to exhibit antitumor activity in PLC both in vitro and in vivo $(18,19)$. The role of DHA in inducing ferroptosis in cancer cells was first reported in head and neck carcinoma cells by Lin et al (20), and later in leukemia cells by Du et al (21) and in glioma cells by Chen et al (22). However, to the best of our knowledge, there is currently no direct evidence outlining the role of DHA in inducing ferroptosis in PLC. Nonetheless, an earlier study from Wang et al (18) reported that DHA increased intracellular ROS levels in LM3 liver cancer cells, suggesting that DHA may trigger ferroptosis in PLC cells.

Cancer cells can thrive under hostile microenvironmental conditions. Within tumor masses, endoplasmic reticulum (ER) stress was reported to be induced by nutrient deprivation, oxygen limitation and a high metabolic demand (23). Malignant cells were discovered to initiate the unfolded protein response (UPR) to cope with the ER stress (24). The ER-resident sensors, eukaryotic translation initiation factor $2 \alpha$ kinase 3 (eIF2), inositol-requiring transmembrane kinase/endoribonuclease $1 \alpha($ IRE1 $\alpha)$ and activating transcription factor (ATF)6, coordinate the UPR if misfolded proteins accumulate and aggregate beyond a tolerable threshold $(23,24)$. The upregulation of ATF4, which is induced by ferroptosis inducers, such as erastin and artesunate, is considered a compensatory effect, as its knockdown was demonstrated to further augment ferroptosis in cancer cells $(16,25)$. In glioma cells, the PERK/ATF4 signaling pathway was activated by DHA and negatively regulated DHA-induced ferroptosis (22). However, to the best of our knowledge, studies investigating the roles of the ATF6- and IRE1 $\alpha$-mediated UPR branches in ferroptosis are scarce and how DHA affects these two UPR mediators remains largely unknown.

In the present study, four PLC cell lines, Hep3B, Huh7, $\mathrm{PLC} / \mathrm{PRF} / 5$ and HepG2, were treated with different concentrations of DHA in the presence or absence of ferroptosis inhibitors or iron ions. To deactivate the UPRs, small interfering RNA was used to knockdown the expression of ATF4, XBP1 or ATF6 in PLC cells.

\section{Materials and methods}

Chemicals.Ferrostatin-1 and DHA were purchased from Shanghai Aladdin Biochemical Technology Co., Ltd. and dissolved in DMSO to final stock concentrations of 5 and $50 \mathrm{mM}$, respectively. Deferoxamine mesylate salt (DFOM; MedChemExpress) and iron chloride hexahydrate (Shanghai Aladdin Biochemical Technology Co., Ltd.) were dissolved in distilled $\mathrm{H}_{2} \mathrm{O}$ to final stock concentrations of $50 \mathrm{mM}$ and $2 \mathrm{mg} / \mathrm{l}$, respectively.

Cell lines and culture. PLC cell lines, Hep3B (p53 null), Huh7 (659 A>G p53 mutant), PLC/PRF/5 (747 G>T p53 mutant) and HepG2 (p53 wild-type), were purchased from The Cell Bank of Type Culture Collection of The Chinese Academy of Sciences. Each cell line underwent short tandem repeat profiling for authentication. All cell lines were free of mycoplasma. Hep3B, PLC/PRF/5 and HepG2 cells were maintained in Minimum Essential Medium Eagle (Gibco; Thermo Fisher Scientific, Inc.) supplemented with 10\% FBS (HyClone; Cytiva), while Huh7 cells were cultured in DMEM (Gibco; Thermo Fisher Scientific, Inc.) supplemented with $10 \%$ FBS. Cells were maintained in an HF-90 cell incubator (KG Medical Industries) in an atmosphere with $5 \% \mathrm{CO}_{2}$ at $37^{\circ} \mathrm{C}$.

Cell transfection. Sequences of small interfering RNA (siRNA/si) ATF4, si-X-box binding protein 1 (XBP1), si-ATF6 or si-Chac glutathione specific $\gamma$-glutamylcyclotransferase 1 (CHAC1) are listed in Table I. The coding region of CHAC1 gene was inserted into a pECMV-3xFLAG-N plasmid for overexpression (Fenghui Biotechnology Co., Ltd.). PLC cells seeded in 6-well plates were transfected with siRNA (100 pM) or pECMV-3xFLAG-N plasmid (vector containing the CHAC1 gene) $(2 \mu \mathrm{g})$ mixed with $6 \mu$ Lipofectamine ${ }^{\circledR} 2000$ (Invitrogen; Thermo Fisher Scientific, Inc.) at $37^{\circ} \mathrm{C}$ for $24 \mathrm{~h}$, then treated with DHA for an additional $24 \mathrm{~h}$.

Cell Counting Kit (CCK)-8 assay. PLC cells were treated with different concentrations of DHA, then the cell viability was determined using a CCK-8 assay (Sigma-Aldrich; Merck KGaA). Briefly, PLC cells were plated into 96-well plates at a density of $3 \times 10^{3}$ cells/well and cultured for $24 \mathrm{~h}$. Following the incubation, the cells were treated with $2,5,7.5,10,20,30,40$ or $50 \mu \mathrm{M}$ DHA for $24 \mathrm{~h}$. Cells were subsequently incubated with $10 \mu \mathrm{l}$ CCK- 8 for $1 \mathrm{~h}$ and the absorbance of each well was measured 
Table I. siRNA sequences.

siRNA Sequence, 5'-3'

\section{siATF4-1}

Forward

Reverse

siATF4-2

Forward

Reverse

siXBP1-1

Forward

Reverse

siXBP1-2

Forward

Reverse

siATF6-1

Forward

Reverse

siATF6-2

Forward

Reverse

siCHAC1-1

Forward

Reverse

siCHAC1-2

Forward

Reverse

si*CHAC1

Forward

Reverse

Negative control

Forward

Reverse
GUCCUCCACUCCAGAUCAUTT AUGAUCUGGAGUGGAGGACTT

UGGAUAUCACUGAAGGAGATT UCUCCUUCAGUGAUAUCCATT

GCAAGUGGUAGAUUUAGAATT UUCUAAAUCUACCACUUGCTT

CCUAAAGUUCUGCUUCUGUTT ACAGAAGCAGAACUUUAGGTT

GAAAUGUCGGUUCAGAUAUTT AUAUCUGAACCGACAUUUCTT

GAGCCACUGAAGGAAGAUATT UAUCUUCCUUCAGUGGCUCTT

GACGCUCCUUGAAGAUCAUTT AUGAUCUUCAAGGAGCGUCTT

GCCACAACCUUGAAUACUUTT AAGUAUUCAAGGUUGUGGCTT

UGGGAAGCUCAUCACUACATT UGUAGUGAUGAGCUUCCCATT

UUCUCCGAACGUGUCACGUTT ACGUGACACGUUCGGAGAATT

siRNA, small interfering RNA; XBP1, X-box binding protein 1; ATF4, activating transcription factor 4; ATF6, activating transcription factor 6; CHAC1, ChaC Glutathione Specific $\gamma$-glutamylcyclotransferase 1.

at a wavelength of $450 \mathrm{~nm}$ using a microplate reader (BioTek Instruments, Inc.). For some experiments, DFOM $(10 \mu \mathrm{M}$ for Huh7 cells and $50 \mu \mathrm{M}$ for the other cell lines), ferrostatin-1 (5 $\mu \mathrm{M}$ for HepG2 cells and $1 \mu \mathrm{M}$ for the other cell lines) or exogenous iron ions (10 $\mu \mathrm{g} / \mathrm{ml}$ iron chloride hexahydrate) were used to treat PLC cells in the presence of DHA.

Establishment of a PLC cellxenograft tumormouse model.A total of 32 male BALB/c nude mice (age, 6-8 weeks; weight, 18-20 g) were obtained from HFK Bioscience Co. Ltd. and housed in a specific pathogen-free facility. The mice were randomly divided into four groups ( $\mathrm{n}=8 /$ group) and PLC cells were subcutaneously injected into the nude mice. The tumor volumes were determined using the following formula: $0.5 \mathrm{x}$ tumor length $\mathrm{x}$ (tumor width) ${ }^{2}$. When the xenografted tumors had grown to $80-100 \mathrm{~mm}^{3}$, half of the mice in each group were administered with $100 \mathrm{mg} / \mathrm{kg}$
DHA for 5 days/week by gavage. After 21 days, all mice were anesthetized with isoflurane (2.5\%; Yuyan) and sacrificed by cervical dislocation immediately $(26,27)$. The present study was approved by the Ethics Committee of Zhengzhou University (Zhengzhou, China).

Hematoxylin and eosin $(H \& E)$. Tumor tissues were collected, fixed with $4 \%$ paraformaldehyde (Xilong Chemicals) overnight at $4^{\circ} \mathrm{C}$, embedded in paraffin and sliced into $5-\mu \mathrm{m}$ thick sections. The sections were subsequently deparaffinized and rehydrated, then incubated with $H \& E$ staining agents (Beijing Solarbio Science \& Technology Co., Ltd.), according to the manufacturer's protocol.

Measurement of ROS levels. Total (Jiancheng Bioengineering Institute) and lipid ROS levels (Invitrogen; Thermo Fisher Scientific, Inc.) were determined using ROS assay kits, according to the manufacturers' protocols, which were based on detecting 2',7'-dichlorofluorescin (DCF) diacetate (cat. no. E004) and C11-BODIPY ${ }^{\circledR}$ 581/591 fluorescence (cat. no. D3861), respectively. The DCF and C11-BODIPY fluorescence intensities were analyzed using a M200 PRO automatic microplate reader (Tecan Group, Ltd.) or a NovoCyte flow cytometer (Agilent Technologies, Inc.), respectively. Raw data from the flow cytometer was analyzed using NovoExpress version 1.4.1 (Agilent Technologies, Inc.).

Measurement of iron concentrations. Intracellular ferrous iron levels were analyzed using an iron assay kit (cat. no. TC1015; Leagene Biotech), according to the manufacturer's protocol. The output was measured on a M200 PRO automatic microplate reader at a wavelength of $562 \mathrm{~nm}$.

Measurement of malondialdehyde (MDA) and glutathione (GSH) levels, and glutathione peroxide (GSH-PX) activity. Kits (all from Jiancheng Bioengineering Institute) were used to determine MDA (cat. no. A003-1) and GSH (cat. no. A061-1) levels, and GSH-PX (cat. no. A005) activity, according to the manufacturers' protocols.

Reverse transcription-quantitative PCR (RT-qPCR). Total RNA was extracted from PLC cells treated with DHA for 1, 6, 12 or $24 \mathrm{~h}$ with a RNAsimple kit (Tiangen Biotech Co., Ltd.). RNAs were reverse transcribed to cDNA using a TIANSeq M-MLV kit (Tiangen Biotech Co., Ltd.) according to the manufacturer's protocol. Briefly, RNAs were incubated in a LifeExpress Classic PCR machine (Hangzhou Bioer Co., Ltd.) at $25^{\circ} \mathrm{C}$ for $10 \mathrm{~min}, 42^{\circ} \mathrm{C}$ for $50 \mathrm{~min}$, and then at $80^{\circ} \mathrm{C}$ for $10 \mathrm{~min}$ to stop the reaction. The splicing of XBP1 mRNA (NM_005080 and NM_001079539) was assessed using qPCR using one pair of primers: Forward, 5'-AAACTTTTGCTAGAAAATCAG C-3' and reverse, 5'-CAATACCGCCAGAATCCA-3', which spanned the splice site. The thermocycling conditions were: $95^{\circ} \mathrm{C}$ for $5 \mathrm{~min}$; followed by 36 of $95^{\circ} \mathrm{C}$ for $10 \mathrm{sec}, 52^{\circ} \mathrm{C}$ for $20 \mathrm{sec}, 72^{\circ} \mathrm{C}$ for $30 \mathrm{sec}$ and $25^{\circ} \mathrm{C}$ for $5 \mathrm{~min}$. This enabled two fragments (252 and $226 \mathrm{bp}$ ) to be detected. The PCR products were subsequently analyzed using a $1.5 \%$ agarose gel.

Western blotting. Total protein was extracted from the whole cell lysates using RIPA lysis buffer (Beijing Solarbio Science 
Table II. Antibodies used for western blot analysis.

\begin{tabular}{|c|c|c|c|}
\hline Primary antibodies & Supplier & Cat. no. & Dilution \\
\hline ATF4 & \multirow[t]{2}{*}{ Cell Signaling Technologies, Inc. } & 11815 & $1: 1,000$ \\
\hline SLC7A11 & & 12691 & $1: 1,000$ \\
\hline ATF6 & \multirow[t]{3}{*}{ ABclonal Biotech Co., Ltd. } & A0202 & $1: 1,000$ \\
\hline p-eIF $2 \alpha$ & & AP0692 & $1: 100$ \\
\hline $\mathrm{eIF} 2 \alpha$ & & A0764 & $1: 500$ \\
\hline GPX4 & \multirow[t]{3}{*}{ Abcam } & AB 125066 & $1: 3,000$ \\
\hline SLC3A2 & & AB 108300 & $1: 5,000$ \\
\hline CHAC1 & & AB76386 & $1: 1,000$ \\
\hline $\mathrm{p}-\mathrm{IRE} 1 \alpha$ & \multirow[t]{3}{*}{ Affinity Biosciences } & DF8322 & $1: 1,000$ \\
\hline IRE1 $\alpha$ & & DF7709 & $1: 1,000$ \\
\hline PERK & & AF5304 & $1: 1,000$ \\
\hline p-PERK & Thermo Fisher Scientific, Inc. & PA5-40294 & $1: 500$ \\
\hline GAPDH & ProteinTech, Group, Inc. & 60004-1-Ig & $1: 10,000$ \\
\hline Histone H3 & Bioss Antibodies & bs-0349R & $1: 1,000$ \\
\hline \multirow[t]{2}{*}{ IgG-horseradish peroxidase } & \multirow[t]{2}{*}{ Beijing Solarbio Science \& Technology Co., Ltd. } & SE131 & $1: 3,000$ \\
\hline & & SE134 & $1: 3,000$ \\
\hline
\end{tabular}

ATF4, activating transcription factor 6; p, phosphorylated; PERK, protein kinase R-like ER kinase; eIF2 $\alpha$, eukaryotic initiation factor $2 \alpha$; IRE1 $\alpha$, inositol-requiring transmembrane kinase/endoribonuclease $1 \alpha$; GPX4, glutathione peroxidase 4; CHAC1, ChaC glutathione specific $\gamma$-glutamylcyclotransferase 1; SLC, solute carrier family.

\& Technology Co., Ltd.) supplemented with $1 \%$ PMSF. Nuclear proteins were isolated using a Nuclear and Cytoplasmic Protein Extraction kit, according to the manufacturer's protocol (Beyotime Institute of Biotechnology). Protein concentration was determined using a BCA assay kit (Beijing Solarbio Science $\&$ Technology Co., Ltd.) and, 10-20 $\mu \mathrm{g}$ protein sample was separated via SDS-PAGE (8-15\%). The resolved proteins were subsequently transferred to PVDF membranes (MilliporeSigma) and blocked with 5\% non-fat milk for $1 \mathrm{~h}$ at room temperature. The membranes were then incubated with primary antibodies (listed in Table II) at $4^{\circ} \mathrm{C}$ overnight. Following primary antibody incubation, the membranes were incubated with secondary antibodies at $37^{\circ} \mathrm{C}$ for $1 \mathrm{~h}$. Protein bands were visualized using ECL reagents (Beijing Solarbio Science \& Technology Co., Ltd.). GAPDH was used as the loading control for whole cell lysates, and Histone $\mathrm{H} 3$ was used as the nuclear loading control.

pGL3 dual luciferase reporter assay. The potential binding sites for ATF4, XBP1 and ATF6 on the CHAC1 gene promoter were predicted using JASPAR (jaspar.genereg.net) and PROMO (alggen.lsi.upc.es/cgi-bin/promo_v3/promo/promoinit. cgi?dirDB=TF_8.3). The promoter $(-2,000$ to $+30 \mathrm{bp})$ of the $\mathrm{CHAC1}$ gene was inserted into a pGL3 luciferase reporter and the promoter activity was determined by analyzing the firefly/ Renilla luciferase ratio, according to the manufacturer's protocol (Promega Corporation).

Statistical analysis. Statistical analysis was performed using GraphPad Prism version 8.0 (GraphPad Software, Inc.) and data are presented as the mean \pm SD. Statistical differences between groups were determined using a one-way or two-way ANOVA followed by a Bonferroni's multiple comparison test.
$\mathrm{P}<0.05$ was considered to indicate a statistically significant difference.

\section{Results}

DHA induced-ferroptosis in PLC cells is irrelevant of p53 status. Considering the involvement of p53 in ferroptosis (25), four PLC cell lines with different p53 statuses, Hep3B (p53 null), Huh7 and PLC/PRF/5 (both p53 mutant) and HepG2 (p53 wild-type), were treated with increasing concentrations of DHA for $24 \mathrm{~h}$. The data revealed that DHA inhibited the survival of all the PLC cell lines, irrelevant of their p53 status (Fig. 1A). Subsequently, two different ferroptosis inhibitors, DFOM (an iron chelator) and ferrostatin-1 (a lipid peroxidation inhibitor) were used to treat PLC cells in the presence of DHA. The results revealed that the cytotoxic effects of DHA on PLC cells were attenuated by both ferroptosis inhibitors (Fig. 1A). Conversely, the addition of exogenous iron ions further augmented the effects of DHA (Fig. 1A).

The $\mathrm{IC}_{50}$ value of DHA was $29.4 \pm 1.7 \mu \mathrm{M}$ for Hep3B cells, $32.1 \pm 4.5 \mu \mathrm{M}$ for Huh7 cells, 22.4 $\pm 3.2 \mu \mathrm{M}$ for PLC/PRF/5 cells and $40.2 \pm 2.1 \mu \mathrm{M}$ for HepG2 cells (Fig. 1B). These findings suggested that HepG2 cells were the most resistant to DHA, followed by Huh7 and Hep3B cells, while PLC/PRF/5 cells were the least resistant. According to the $\mathrm{IC}_{50}$ values, HepG2, Huh7, Hep3B and PLC/PRF/5 cells were further treated with $40,35,30$ or $25 \mu \mathrm{M}$ DHA, respectively.

Following incubation with DHA for 1, 6, 12 or $24 \mathrm{~h}$, PLC cells were harvested to analyze the total and lipid ROS contents (Fig. 1C and D), MDA levels (Fig. 1E) and iron concentrations (Fig. 1F). The results revealed that the ROS, MDA and iron levels were increased in PLC cells exposed to DHA. These 
A
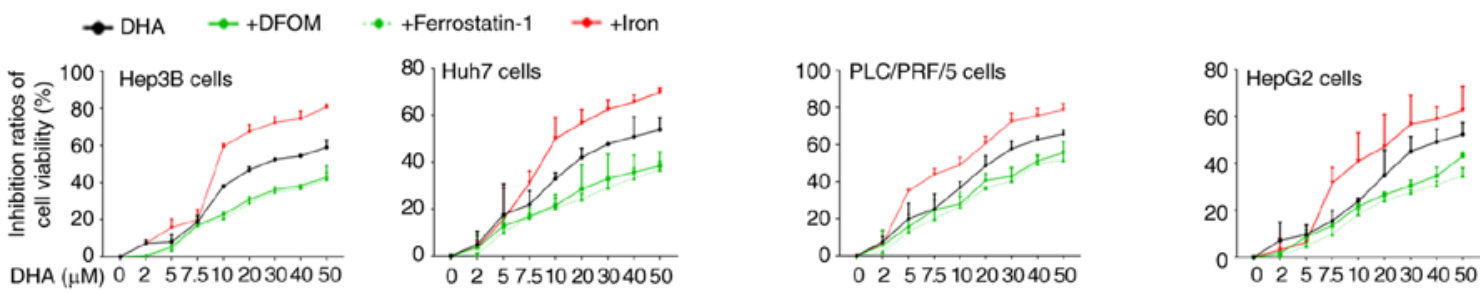

B
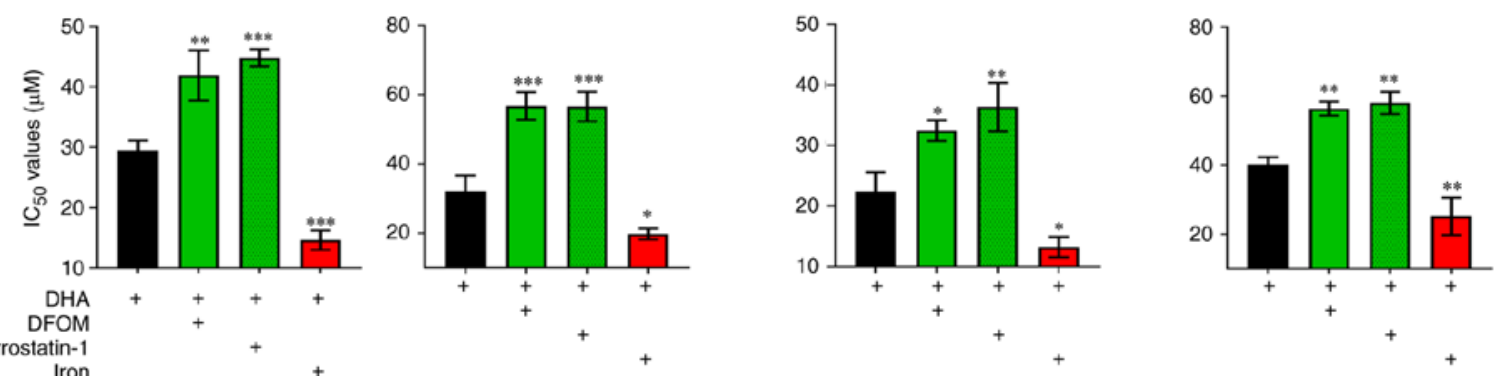

C
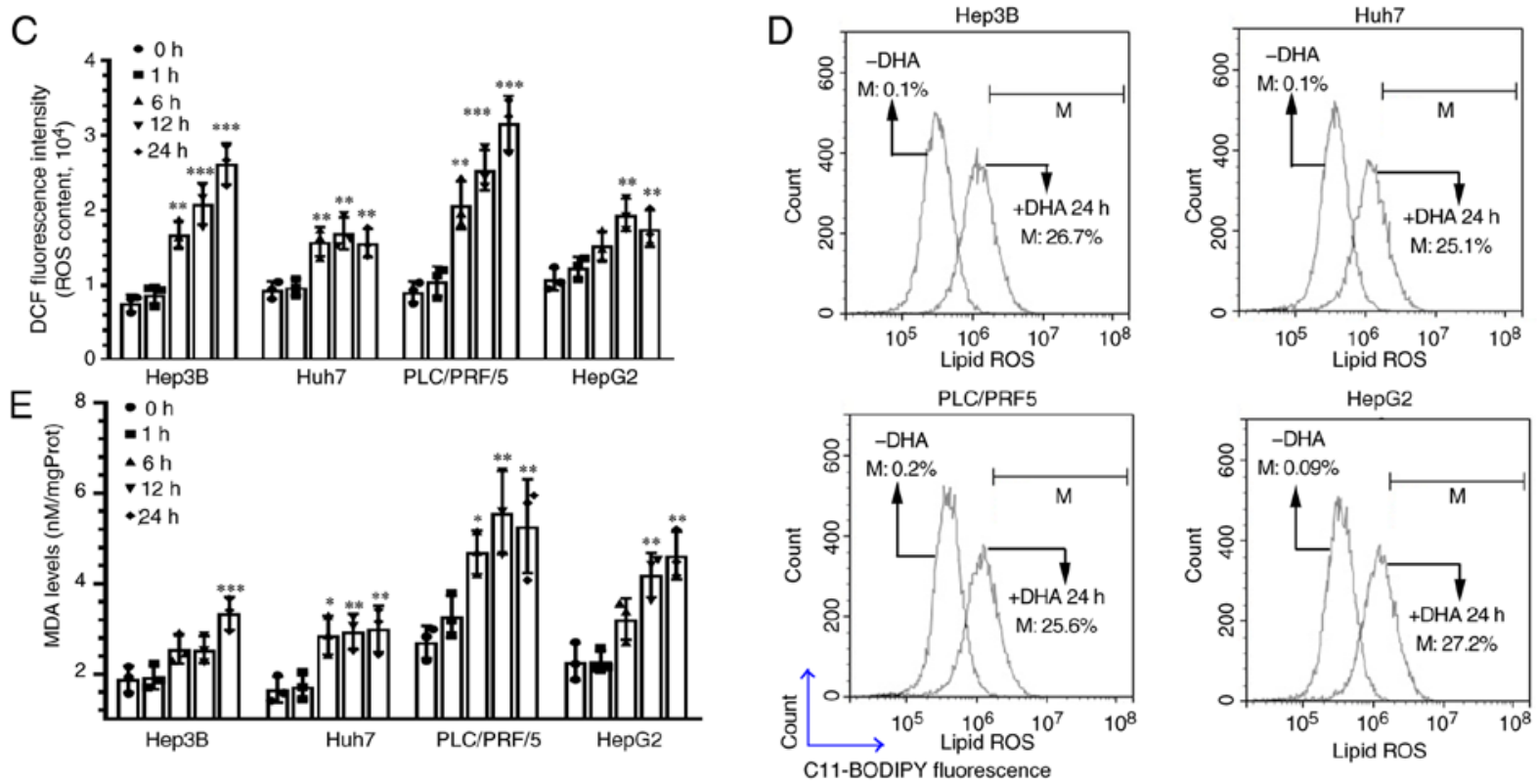

$\mathrm{F}$
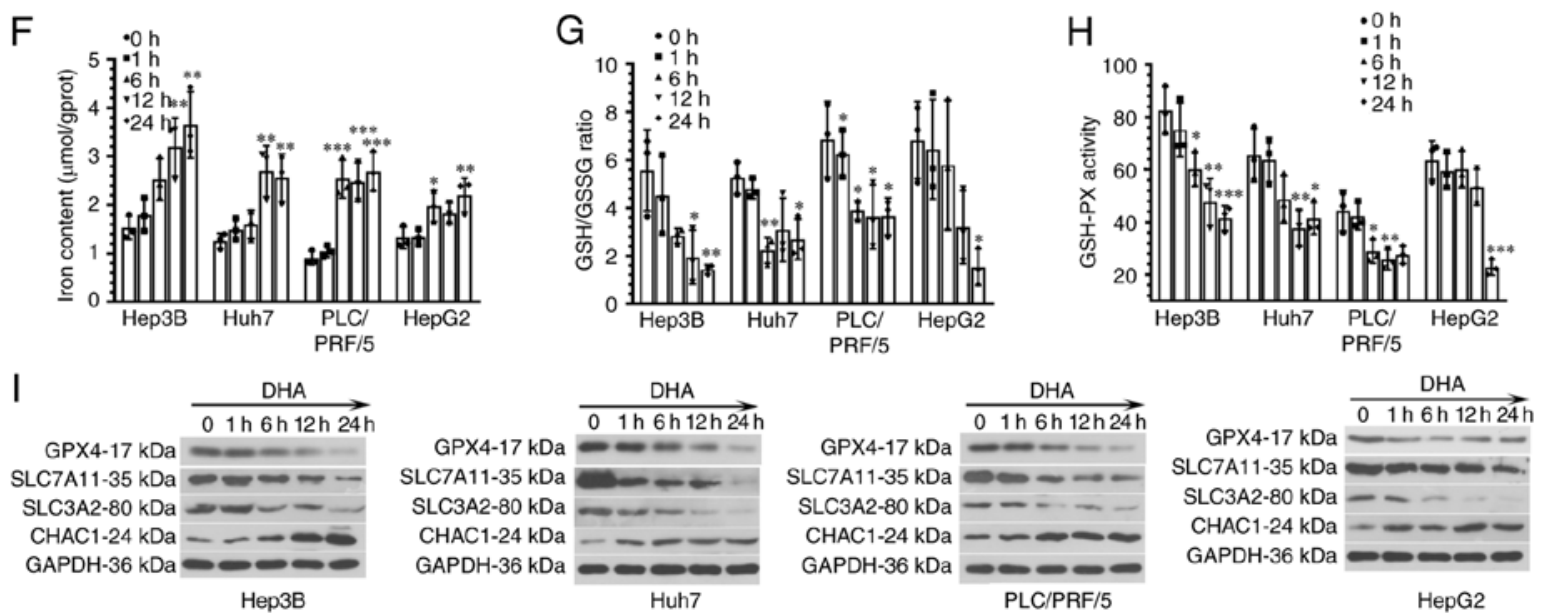

Figure 1. DHA induces ferroptosis in PLC cells in vitro. (A) Hep3B (p53 null), Huh7 and PLC/PRF/5 (both p53 mutant) and HepG2 (p53 wild-type) cells were treated with increasing concentrations of DHA for $24 \mathrm{~h}$. Two different ferroptosis inhibitors, DFOM (an iron chelator; $10 \mu \mathrm{M}$ for Huh7 cells and $50 \mu \mathrm{M}$ for the other cell lines) and ferrostatin-1 (a lipid peroxidation inhibitor; $5 \mu \mathrm{M}$ for HepG2 and $1 \mu \mathrm{M}$ for the other cell lines) were used to treat PLC cells in the presence of DHA. Iron chloride hexahydrate $(10 \mu \mathrm{g} / \mathrm{ml})$ was used to increase the iron concentration. The vitality of PLC cells were determined using Cell Counting Kit- 8 assays. (B) $\mathrm{IC}_{50}$ values were calculated. (C) Based on the $\mathrm{IC}_{50}$ values, HepG2, Huh7, Hep3B and PLC/PRF/5 were treated with 40,35, 30 and $25 \mu \mathrm{M}$ DHA, respectively. After incubation with DHA for 1, 6, 12 or $24 \mathrm{~h}$, (C) total ROS, (D) lipid ROS generation. (E) MDA levels, (F) iron concentrations, (G) GSH/GSSG ratios (H) and GSH-PX activity, as well as (I) the protein expression levels of GPX4, SLC7A11, SLC3A2 and CHAC1 in PLC cells were determined. Data are presented as the mean \pm standard deviation of three repeats. ${ }^{*} \mathrm{P}<0.05,{ }^{* * *} \mathrm{P}<0.01,{ }^{* * *} \mathrm{P}<0.001$ vs. control ( 0 h). DHA, dihydroartemisinin; PLC, primary liver cancer; DFOM, deferoxamine mesylate salt; ROS, reactive oxygen species; MDA, malondialdehyde; GSSG, oxidized glutathione; GSH-PX, glutathione-peroxidase; GPX4, glutathione peroxidase 4; SLC, solute carrier family; CHAC, ChaC glutathione specific $\gamma$-glutamylcyclotransferase; DCF, dichlorofluorescin; M, \% of control. 
A

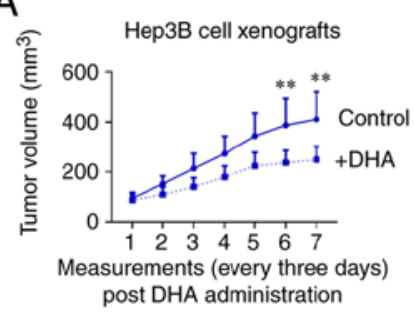

B

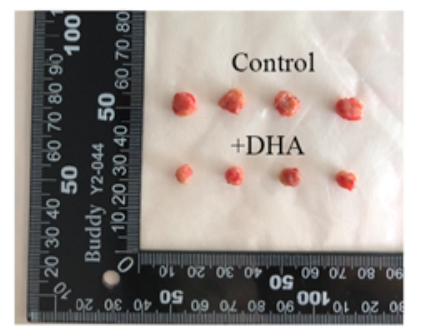

Hep3B cell xenografts

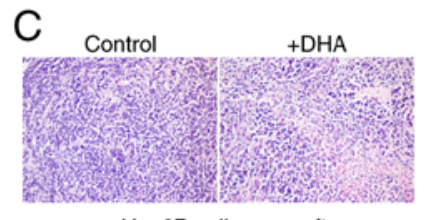

Hep3B cell xenografts

\section{D}

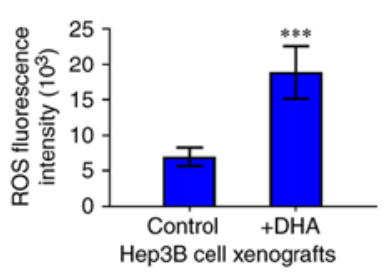

E

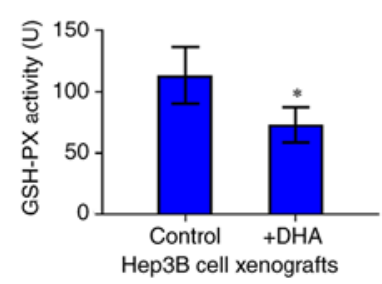

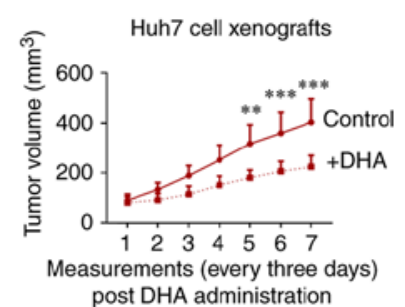

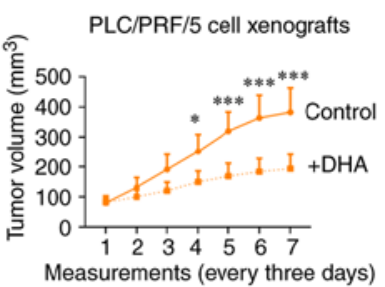
post DHA administration

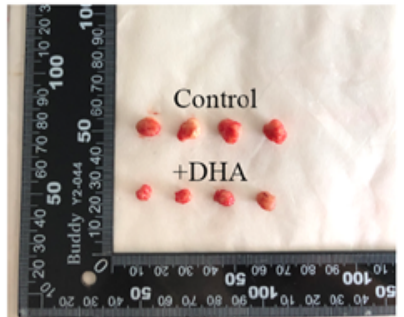

Huh7 cell xenografts

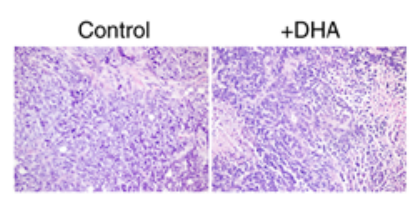

Huh7 cell xenografts
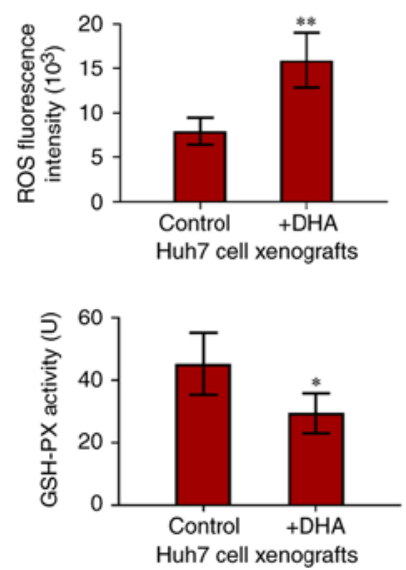

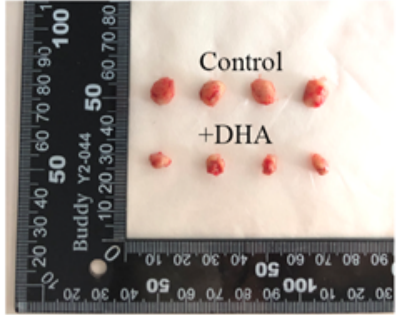

PLC/PRF/5 cell xenografts

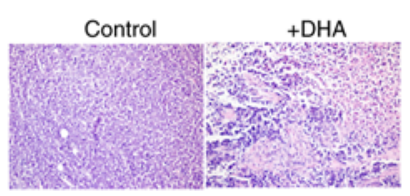

PLC/PRF/5 cell xenografts
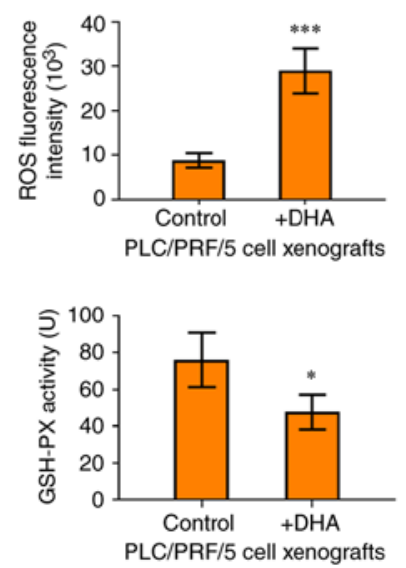
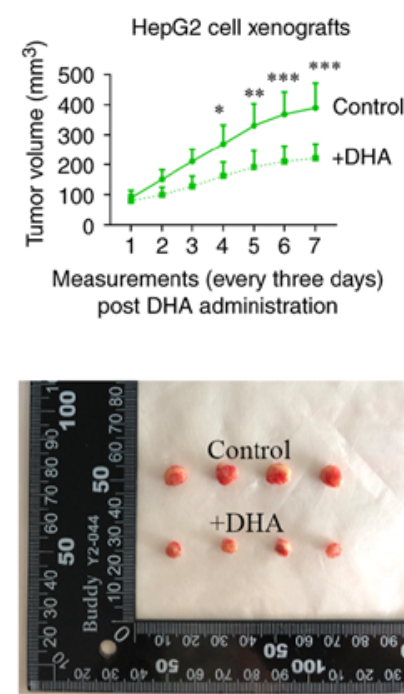

HepG2 cell xenografts

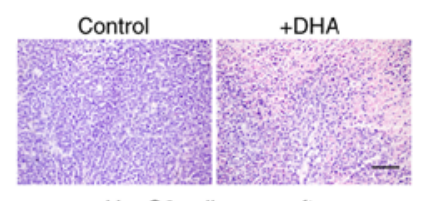

HepG2 cell xenografts
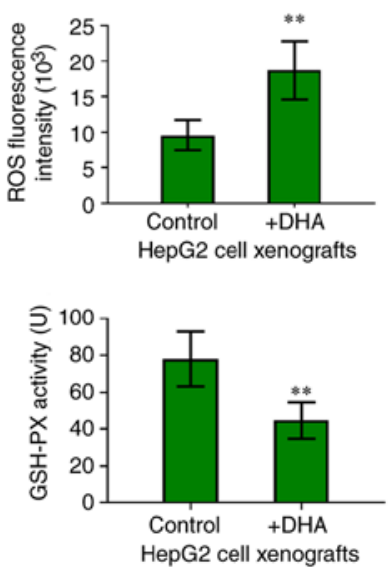

Figure 2. DHA reduces PLC tumor growth in nude mice. (A and B) PLC cells were subcutaneously injected into immune deficient mice. DHA (100 mg/kg/day, 5 days/week) was administered to mice when tumor volumes reached $80-100 \mathrm{~mm}^{3}$. The tumor volumes were recorded every 3 days, and (C) 3 weeks later, the xenografted tumors were collected for hematoxylin and eosin staining (scale bar, $100 \mu \mathrm{m}$ ). (D) ROS and (E) GSH-PX activities in tumor tissues were determined. Data are presented as the mean \pm standard deviation of four repeats. ${ }^{*} \mathrm{P}<0.05,{ }^{* *} \mathrm{P}<0.01,{ }^{* * *} \mathrm{P}<0.001 \mathrm{vs}$. control $(0 \mathrm{~h})$. DHA, dihydroartemisinin; PLC, primary liver cancer; ROS, reactive oxygen species; GSH-PX, glutathione-peroxidase.

data suggested that DHA may act as a ferroptosis inducer in PLC cells in vitro.

Blocking GSH synthesis is known to facilitate the accumulation of toxic ROS (7). Therefore, the content of GSH in DHA-treated PLC cells was analyzed using a commercial kit. Compared with the blank cells, the GSH/glutathione disulfide (oxidized form of GSH) ratio was decreased in cells exposed to DHA (Fig. 1G). The expression levels of GPX4, SLC7A11 and SLC3A2, and the activity of GSH-PX were also inhibited by DHA treatment (Fig. $1 \mathrm{H}$ and I). Conversely, the expression levels of $\mathrm{CHAC} 1$ were upregulated in response to DHA treatment (Fig. 1I). These data suggested that DHA may act as a negative regulator of GSH synthesis in PLC cells.
DHA limits PLC xenograft tumor growth in vivo. The effects of DHA on PLC growth in vivo were further investigated by subcutaneously injecting PLC cells into immune-deficient mice; no weight loss or health problems were observed in the mice. DHA was then administered to the mice upon the tumor volumes reaching $80-100 \mathrm{~mm}^{3}$. The tumor volumes were recorded every 3 days and 3 weeks later, the xenografted tumors were collected. As shown in Fig. 2A-C, DHA limited, but did not completely inhibit, the formation of PLC xenograft tumors. Furthermore, DHA increased ROS accumulation (Fig. 2D) and decreased GSH-PX activity (Fig. 2E) in the tumor masses. These data, together with the aforementioned results from the in vitro experiments, indicated that DHA may induce ferroptosis both in vitro and in vivo. 
A

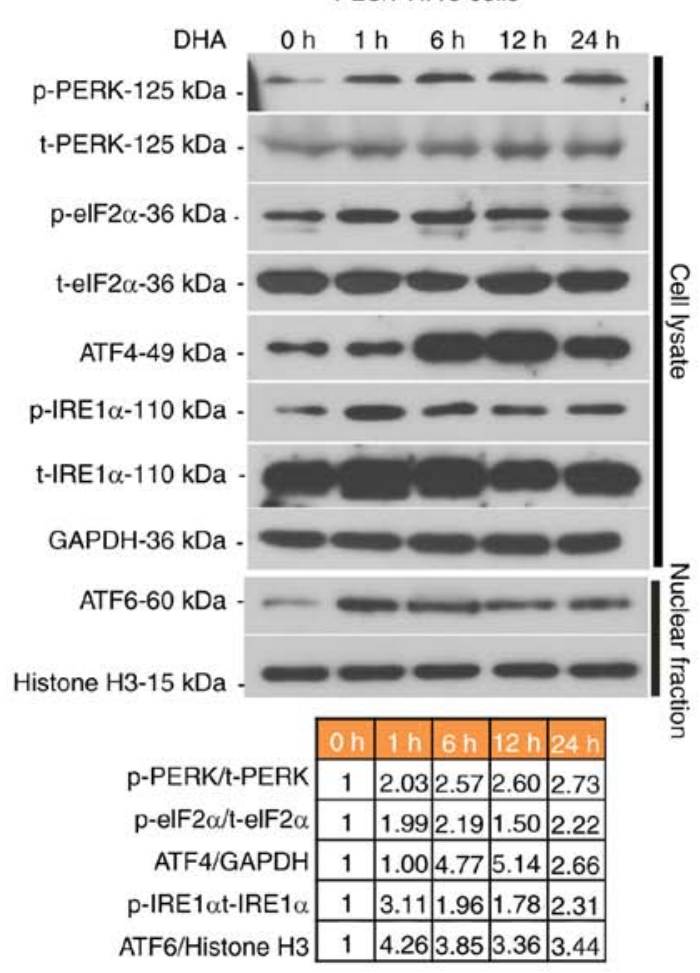

B

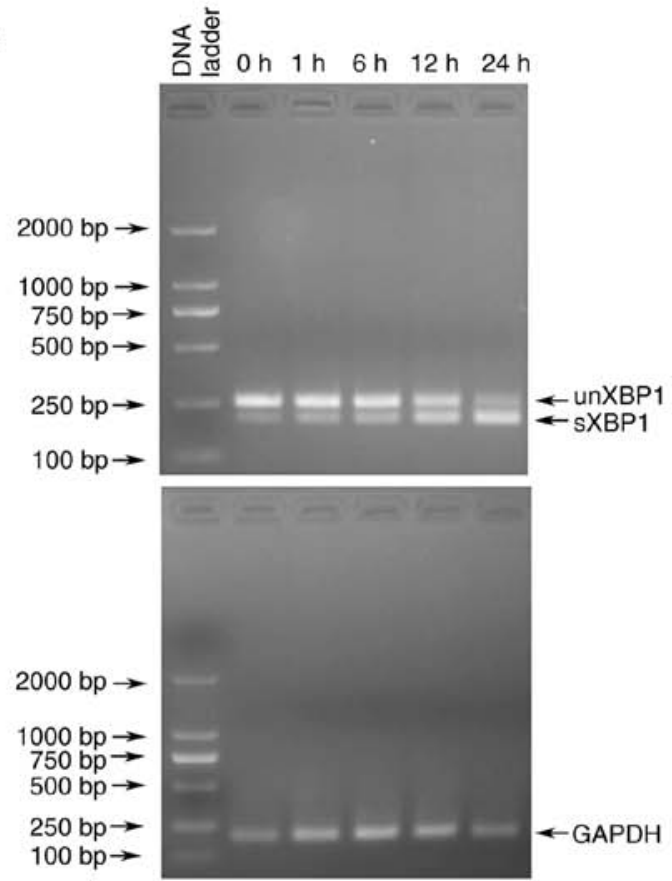

C

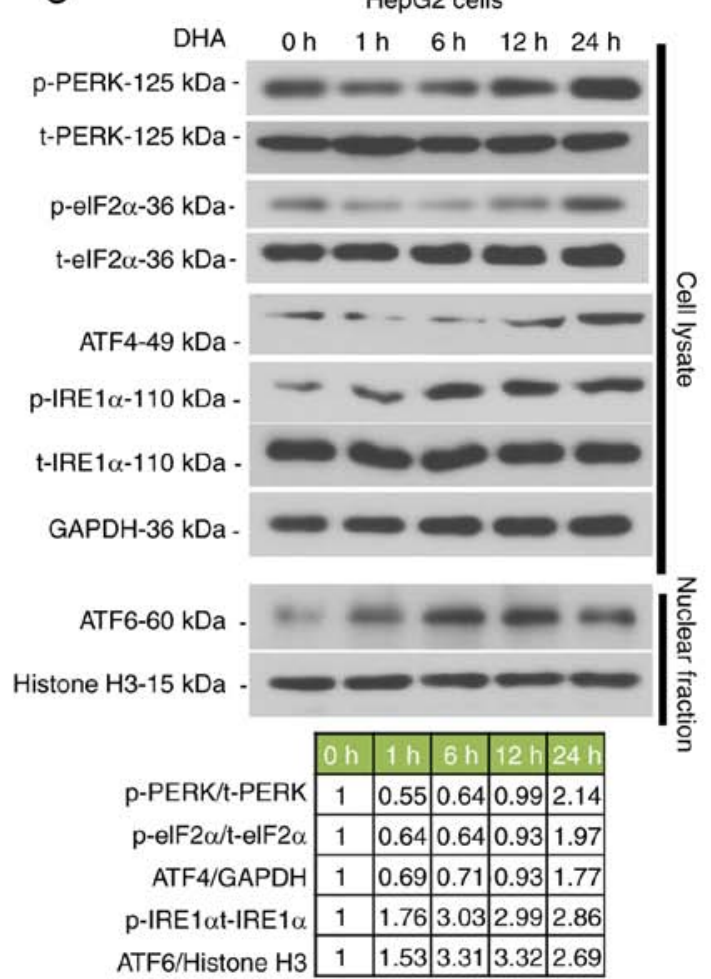

D
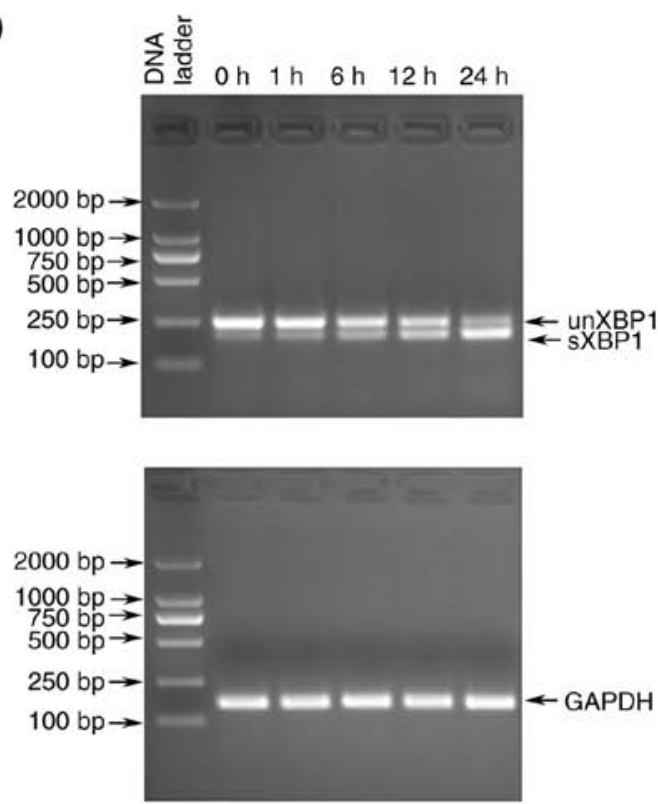

Figure 3. DHA activates the UPRs in PLC cells in vitro. HepG2 and PLC/PRF/5 cells were treated with 40 and $25 \mu \mathrm{M}$ DHA, respectively, for $1,6,12$ or $24 \mathrm{~h}$. (A and C) The protein expression levels of UPR-associated molecules were assessed by western blotting. Relative protein expression levels of indicated molecules are listed below the blots. (B and D) Total RNAs were isolated to analyze the formation of sXBP1 in PLC cells. DHA, dihydroartemisinin; PLC, primary liver cancer; UPR, unfolded protein response; p-, phosphorylated; t-, total; PERK, protein kinase R-like ER kinase; eIF2 $\alpha$, eukaryotic initiation factor $2 \alpha$; IRE1 $\alpha$, inositol-requiring transmembrane kinase/endoribonuclease $1 \alpha$; ATF, activating transcription factor.

DHA activates the three branches of the UPR in PLC cells. As DHA induced ferroptosis in all the analyzed PLC cells both in vivo and in vitro, alterations in UPR-associated molecules were next investigated in PLC/PRF/5 and HepG2 cells. The data demonstrated that all three branches of the UPR signaling pathways were activated by DHA, which was evidenced through the upregulated expression levels of phosphorylated PERK, eukaryotic translation initiation factor $2 \mathrm{~A}$ (eIF2 $\alpha$ ), IRE1 $\alpha$, ATF4, nuclear ATF6 and spliced XBP1 (Fig. 3A-D). Notably, DHA was found to activate PERK/eIF2 $\alpha /$ ATF4 signaling earlier in PLC/PRF/5 cells than in HepG2 cells (Fig. 3A-D). To determine the role of the activated UPR in DHA-mediated ferroptosis, ATF4, XBP1 and ATF6 were silenced with their respective siRNAs (Fig. 4A and E). The transfection efficiencies 


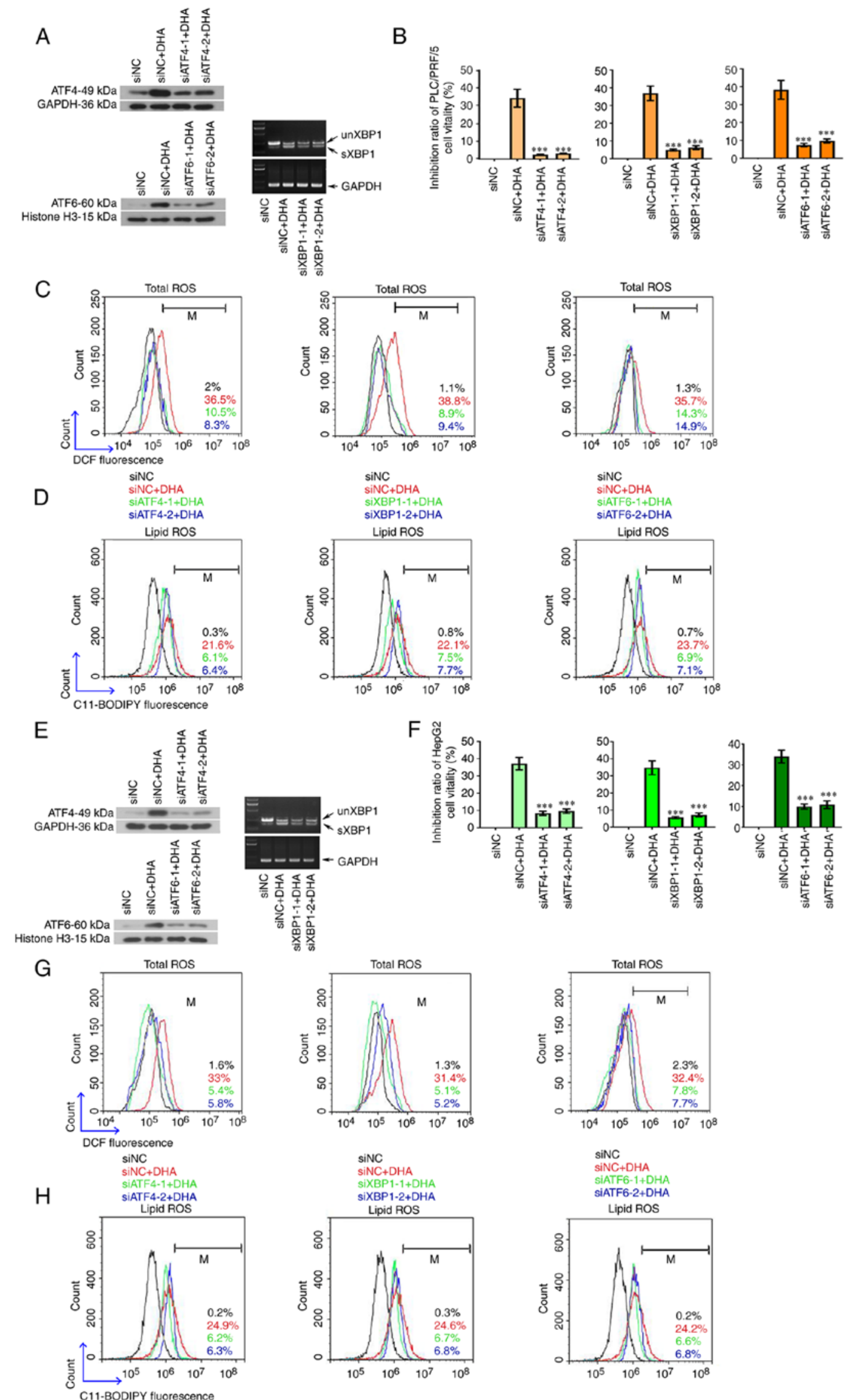

Figure 4. The cytotoxic effects of DHA are attenuated following suppression of the UPR. (A and E) Specific siRNAs were synthesized to target ATF4, XBP1 and ATF6 in PLC cells. A total of $24 \mathrm{~h}$ post siRNA transfection, HepG2 and PLC/PRF/5 cells were treated with 40 or $25 \mu \mathrm{M}$ DHA, respectively, for $24 \mathrm{~h}$. (B and F) Cell viability was determined using a Cell Counting Kit-8, and (C and G) intracellular total ROS and (D and H) lipid ROS levels were determined by flow cytometry. Data are presented as the mean \pm standard deviation of three repeats. ${ }^{* * * *} \mathrm{P}<0.001 \mathrm{vs.} \mathrm{DHA}+$ siNC. DHA, dihydroartemisinin; UPR, unfolded protein response; siRNA, small interfering RNA; ATF, activating transcription factor; XBP1, X box-binding protein 1; sXBP1, spliced form of XBP1; unXBP1, unspliced form of XBP1; PLC, primary liver cancer; ROS, reactive oxygen species; NC, negative control; M, \% of control. 
A

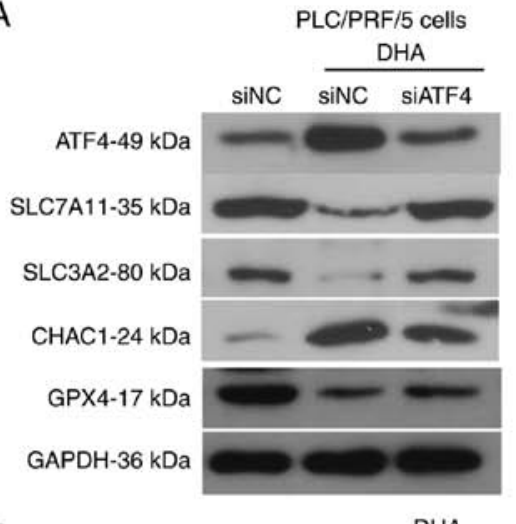

B

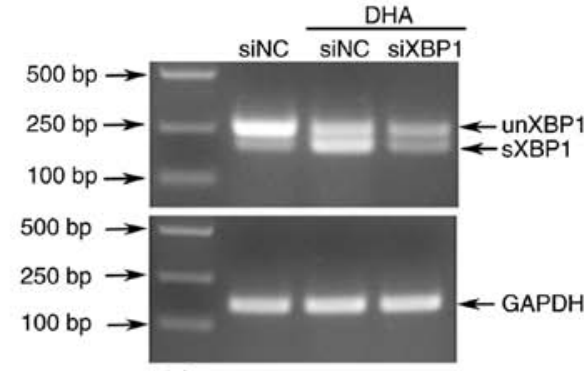

芝弯

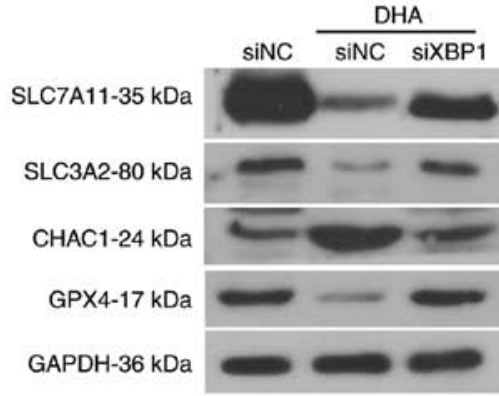

C

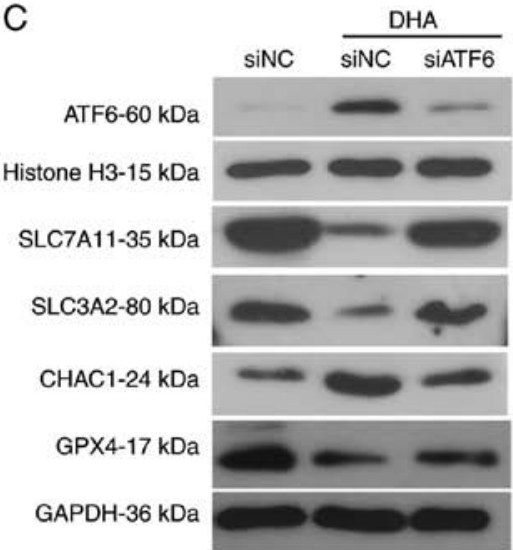

D

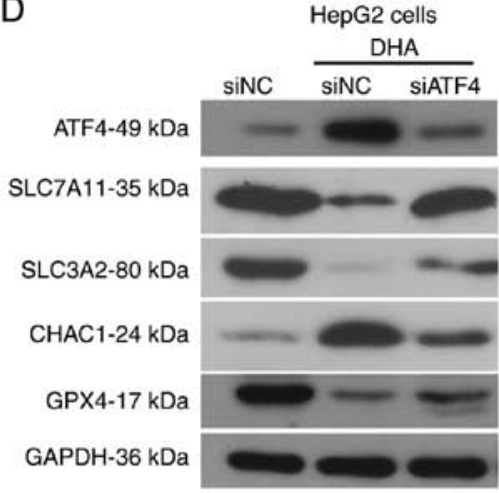

E

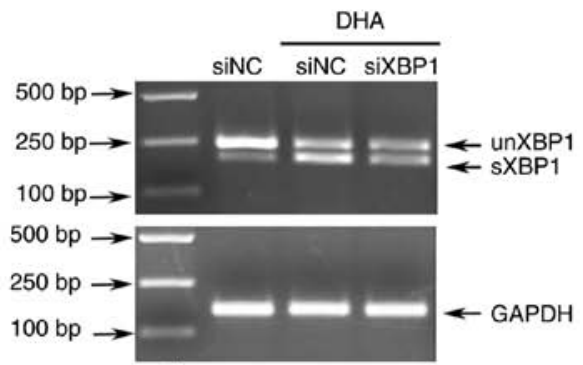

芒㙜

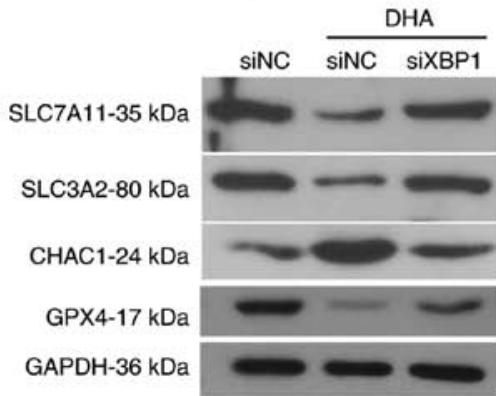

F

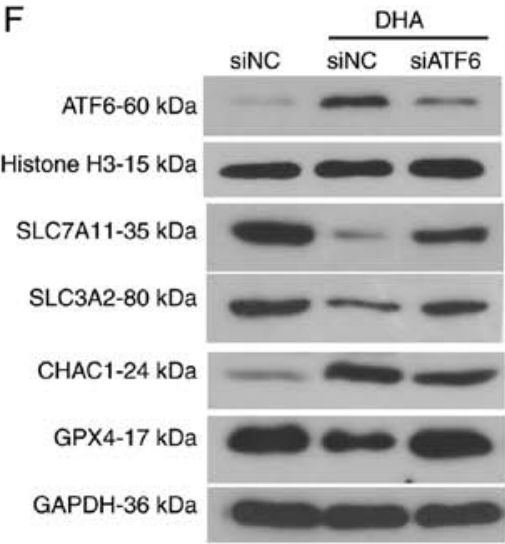

Figure 5. Knockdown of UPR proteins partly restores the expression of molecules associated with ferroptosis in PLC cells in the presence of DHA. (A-F) Specific siRNAs were synthesized to target ATF4, XBP1 and ATF6 in PLC cells. A total of $24 \mathrm{~h}$ post-siRNA transfection, HepG2 and PLC/PRF/5 were treated with 40 or $25 \mu \mathrm{M}$ DHA, respectively, for $24 \mathrm{~h}$. Cellular proteins were extracted to analyze the protein expression levels of proteins associated with the UPR via western blot analysis. (B and E) Total RNAs were isolated to analyze the mRNA expression levels sXBP1 in PLC cells. siRNA, small interfering RNA; ATF, activating transcription factor; XBP1, X box-binding protein 1; sXBP1, spliced form of XBP1; unXBP1, unspliced form of XBP1; PLC, primary liver cancer; UPR, unfolded protein response; NC, negative control; GPX4, glutathione peroxidase 4; SLC, solute carrier family; CHAC, ChaC glutathione specific $\gamma$-glutamylcyclotransferase.

of siRNAs were first determined in PLC cells via western blotting or RT-PCR analysis (Fig. S1). Following the knockdown of all three UPR sensors, PLC cell viability increased (Fig. 4B and F), and total (Fig. 4C and G) and lipid ROS (Fig. 4D and H) content was decreased. The expression levels of GPX4,
SLC7A11, SCL3A2 and CHAC1 were restored to different degrees in cells following the knockdown of the three UPR transcription factors (Fig. 5A-F). To the best of our knowledge, these data indicated, for the first time, that DHA may activate the UPR as an anti-survival mechanism in PLC cells. 
A

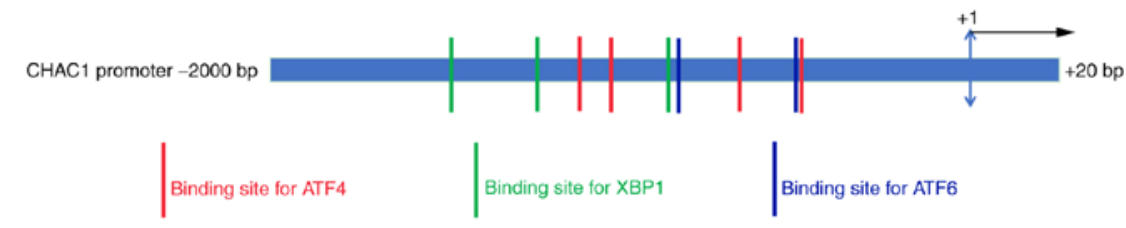

B
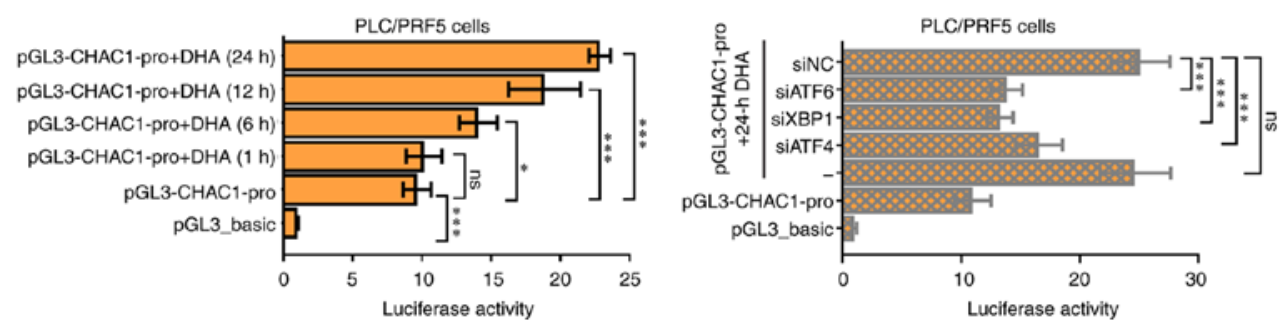

C

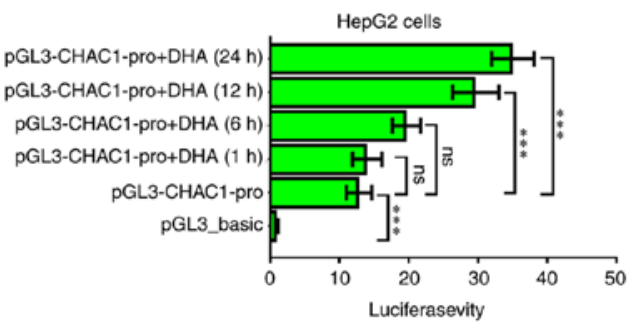

D

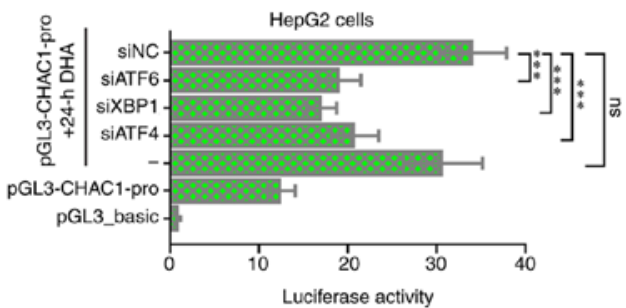

PLC/PRF5 cells

E

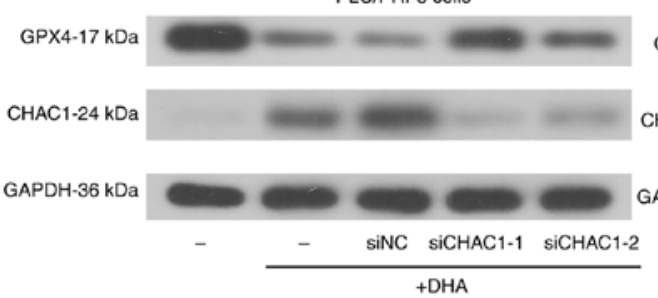

E HepG2 cells

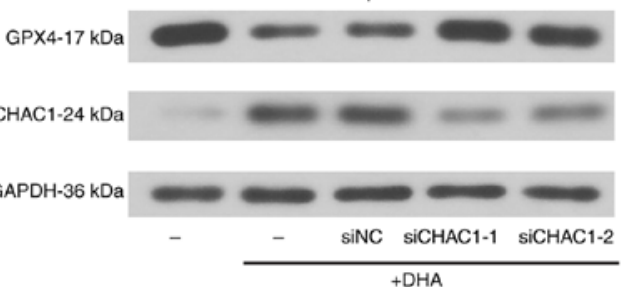

$\mathrm{F}$
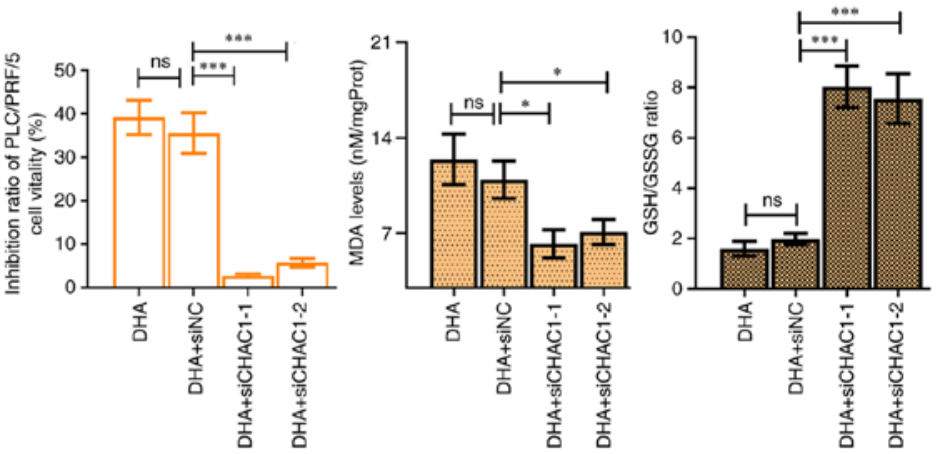

$\mathrm{H}$

G
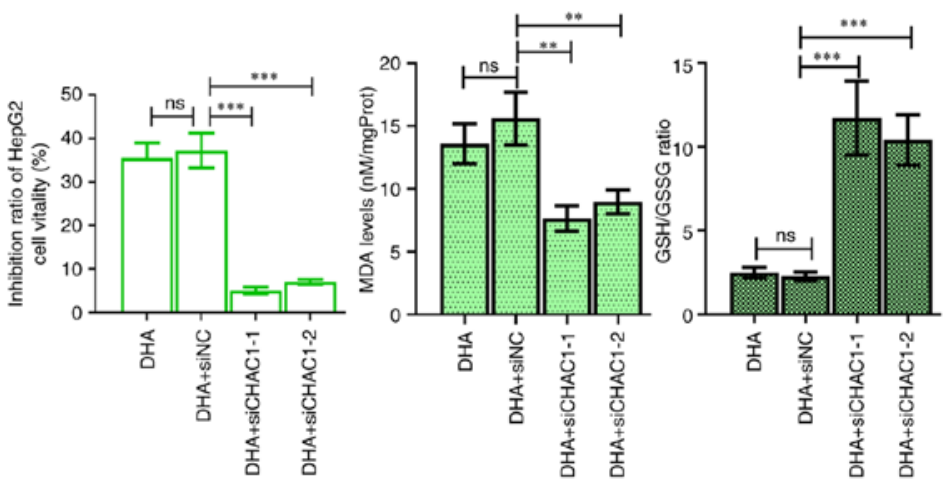

।

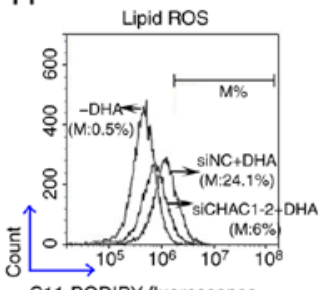

C11-BODIPY fluorescence

Figure 6. DHA enhances the promoter activity of the CHAC1 gene. (A) Potential binding sites for ATF4, XBP1 and ATF6 on the CHAC1 gene promoter (B and C) pGL3 luciferase reporter assay was performed to determine the promoter activity of CHAC1. To knockdown CHAC1 expression, 2 siRNAs were synthesized to target CHACl in PLC cells. A total of $24 \mathrm{~h}$ post siRNA transfection, HepG2 and PLC/PRF/ 5 cells were treated with 40 or $25 \mu \mathrm{M} \mathrm{DHA}$, respectively for $24 \mathrm{~h}$. (D and E) Protein expression levels of GPX4 and CHAC1 were determined by western blotting. (F and G) Cell viability, MDA levels and GSH/GSSG ratios, as well as (H and I) intracellular lipid ROS levels were determined. Data are presented as the mean \pm standard deviation of three repeats. ${ }^{*} \mathrm{P}<0.05$, ${ }^{* *} \mathrm{P}<0.01,{ }^{* * *} \mathrm{P}<0.001$ vs. control. DHA, dihydroartemisinin; PLC, primary liver cancer; CHAC1, ChaC glutathione specific $\gamma$-glutamylcyclotransferase 1; ATF, activating transcription factor; XBP1, X box-binding protein 1; siRNA, small interfering RNA; GPX4, glutathione peroxidase 4; ROS, reactive oxygen species; MDA, malondialdehyde; GSSG, oxidized glutathione; siRNA, small interfering RNA; NC, negative control; M, \% of control. 
A

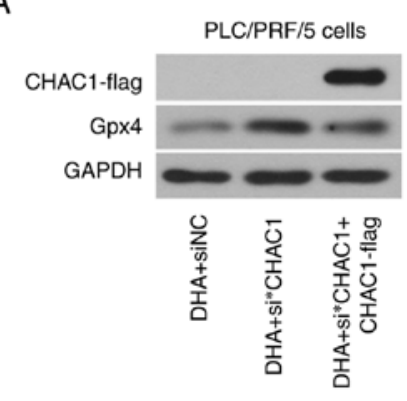

D

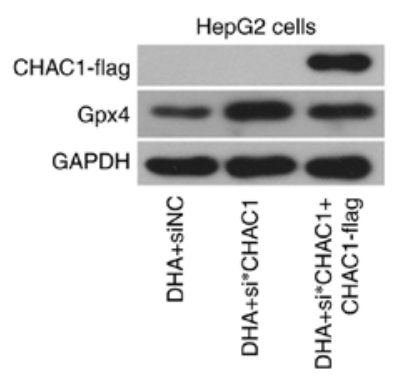

B

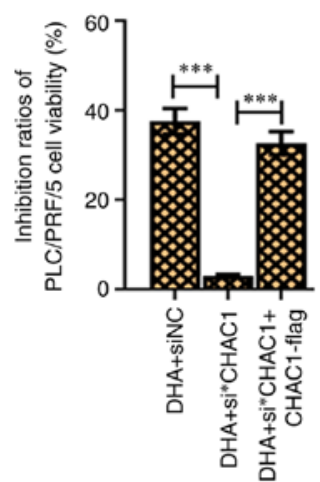

E

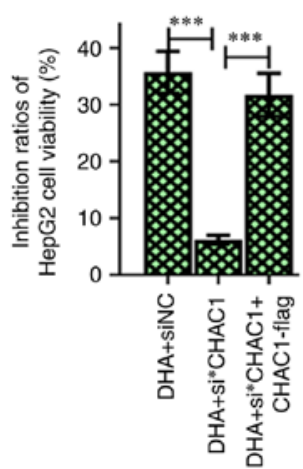

C

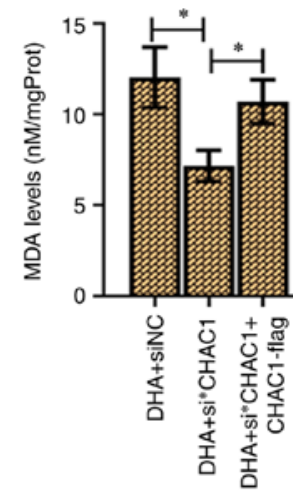

F

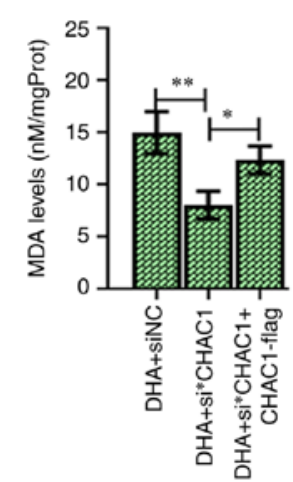

Figure 7. Overexpression of CHAC1 sensitizes PLC cells to DHA. Si*CHAC1 exclusively targeting the 3' untranslated region was used to knockdown endogenous CHAC1 expression. (A and D) Knockdown of CHAC1 was assessed using western blotting. (B and E) Cell viability and (C and F) MDA levels were determined. Data are presented as the mean \pm standard deviation of four repeats. ${ }^{*} \mathrm{P}<0.05,{ }^{* * *} \mathrm{P}<0.01,{ }^{* * *} \mathrm{P}<0.001$ vs. control. DHA, dihydroartemisinin; siRNA, small interfering RNA; NC, negative control; CHAC1, ChaC glutathione specific $\gamma$-glutamylcyclotransferase 1; PLC, primary liver cancer; MDA, malondialdehyde.

DHA promotes the transcription of CHACl by activating the UPRs in PLC cells. Multiple binding sites for the transcription factors were found on the $\mathrm{CHAC1}$ promoter (Fig. 6A). Since DHA could effectively upregulate the expression of ATF4, XBP1 and ATF6, it was hypothesized that this agent may promote CHAC1 transcription. The results from the pGL3 dual luciferase reporter assay validated that DHA enhanced the promoter activity of the CHAC1 gene (Fig. 6B and $\mathrm{C}$, left panel), and that this effect was suppressed following knockdown of ATF4, XBP1 or ATF6 (Fig. 6B and C, right panel). To further validate the role of $\mathrm{CHAC} 1$ in DHA-induced ferroptosis, siRNA was used to knockdown CHAC1 expression (Fig. 6D and E). The results revealed that DHA-induced ferroptosis was suppressed by siCHAC1 (Fig. 6F-I). CHAC1 encoding fragment with a lack of the 3'UTR was inserted into an expression vector, which resulted in a lack of effect of the siRNA targeting CHAC1 3'UTR (si*CHAC1) on its expression. Additionally, it was further found that overexpression of CHAC1 sensitized PLC cells to DHA (Fig. 7).

\section{Discussion}

The induction of ferroptosis is currently being investigated as an alternative approach to eradicate apoptosis-resistant cancer cells (11). Several artemisinin derivates, such as artesunate, artemether and arteether, have been demonstrated to kill cancer cells by inducing ferroptosis (15). In addition, previous studies have revealed that DHA acts as a ferroptosis inducer in cancer cells (20-22). Consistent with the findings of these previous studies, the present study demonstrated that DHA killed PLC cells by inducing ferroptosis.

The classic method to determine whether a drug can induce ferroptosis is to co-treat cancer cells with ferroptosis blockers or iron ions $(12,13)$. The antitumor effects of ferroptosis inducers, such erastin and sorafenib, were found to be attenuated by iron chelators (such as DFOM) and inhibitors of lipid peroxidation inhibitors (such as ferrostatin-1), but augmented following the addition of exogenous iron ions $(7,28)$. In the current study, based on the $\mathrm{IC}_{50}$ results, it was suggested that PLC cells were more resistant to DHA-induced cytotoxicity when ferroptosis was suppressed by DFOM or ferrostatin-1. In contrast, the addition of iron chloride hexahydrate sensitized PLC cells to DHA. Of note, treatment with DFOM or ferrostatin-1 did not completely abolish the antitumor effects of DHA, suggesting that triggering ferroptosis may not be the only method through which DHA can induce cytotoxicity in PLC cells. Although DHA has been shown to induce ferroptosis in other types of cancer cells (20-22), the present study was the first to demonstrate such effects in PLC cells, to the best of our knowledge.

p53 is a key tumor suppressor gene, and mutations in the gene or its loss of function is known to promote tumorigenesis (29). SLC7A11 and SLC3A2 control the import of extracellular cysteine and cystine, and regulate GSH synthesis $(9,30)$. Notably, both wild-type and acetylation-defective mutant p53 were discovered to downregulate SLC7A11 expression, thereby inhibiting GSH synthesis and sensitizing tumor cells to ferroptosis (31). In light of the key role of p53 
in SLC7A11-associated ferroptosis, the present study aimed to investigate the cytotoxicity of DHA in four PLC cell lines with different p53 statuses. The current data demonstrated that DHA could effectively induce ferroptosis in the analyzed PLC cells, even in the p53 null cells (Hep3B cells). It is worth noting that DHA also exhibited antitumor activity against PLC by activating caspase 3 , a key apoptosis regulator, regardless of the p53 status (32). This finding, together with the results of the present study, suggested that DHA may exert its antitumor effects in a p53-independent manner. Thus, DHA may represent an attractive drug for treating p53-mutant or -deficient cancers.

GPX4 was first identified by Ursini et al (33) in 1982 and it has since been shown to reduce reactive phosphatidylcholine hydroperoxides and suppress lipid peroxidation (8). GSH depletion was found to downregulate GPX4 expression (34). In cancer cells, ferroptosis was shown to be induced by GPX4 inactivation (11). To further explore how DHA induces ferroptosis in PLC cells, GSH content and the expression and activity of GPX4 were analyzed. The present data revealed that DHA reduced GSH synthesis and downregulated GPX4 expression in PLC cells. The decreased activity of GPX4 observed in PLC cells exposed to DHA may result from the downregulated expression of GPX4. While a study by Lin et al (20) supported the findings of the present study, a recent study from Chen et al (22) did not report consistent results. The latter study demonstrated that DHA induced ferroptosis in glioma cells and upregulated GPX4 expression in U251 and U373 cancer cells as a compensatory mechanism (22). At present, a reasonable explanation for such paradoxical findings has not been found. Nonetheless, these findings suggested that the effects of DHA on regulating GPX4 expression in different solid tumor types may be inconsistent, and further investigations into the underlying mechanisms are warranted.

The present study also sought to determine whether the DHA-activated UPR played a role in ferroptosis. The UPR is coordinated by three branches, namely, the PERK/eIF2 $\alpha / A T F 4$, IRE1 $\alpha / \mathrm{XBP} 1$ and ATF6 branches $(23,24)$. In response to ER stress, PERK is self-phosphorylated to induce the phosphorylation of eIF2 $\alpha$, thereby promoting ATF4 translation (35). Once phosphorylated, IRE1 $\alpha$ splices XBP1 into the short form (35). ATF6 translocates into the cell nucleus to function as a transcription factor after cleavage from the ER (36). ATF4 was suggested to protect against ferroptosis due to its ability to activate the transcription of SLC7A11 (25). Our previous study also demonstrated that ATF4 acted as a pro-survival factor in erastin-induced ferroptosis in PLC cells (37). Therefore, it was hypothesized that blocking the PERK/eIF2 $\alpha /$ ATF4 signaling pathway may further augment the antitumor effects of DHA in PLC cells. However, by determining the cell viability and ROS contents, the results found that the knockdown of ATF4 attenuated the cytotoxic effects of DHA. Notably, DHA-induced ferroptosis was attenuated following the knockdown of XBP1 or ATF6. Unlike the majority of previous studies revealing the anti-ferroptotic roles of these UPR proteins $(22,25)$, the findings of the present study suggested that the UPR proteins acted as pro-ferroptosis molecules in DHA-treated PLC cells.

Due to the observed role of ATF4 in inducing SLC7A11 expression (25), little previous evidence supports the pro-ferroptotic role of ATF4 observed in the current study.
Nonetheless, an earlier study evaluating the effects of artesunate, another classic ferroptosis inducer, in Burkitt's lymphoma cells indirectly supported the findings of the present study (16). A previous study showed that ATF4 activated the transcription of $\mathrm{CHAC} 1$ to augment artesunate-induced ferroptosis in DAUDI and CA-46 cells (16). CHAC1 was found to function as a $\gamma$-glutamyl cyclotransferase to induce GSH degradation (38). These findings suggested that ATF4 upregulation may also lead to GSH degradation. It is possible that DHA, like artesunate, activates mechanisms important for GSH degradation, which are mediated by UPR proteins, rather than GSH synthesis. In the present study, the results of the dual luciferase reporter assay demonstrated that DHA enhanced the promoter activity of the CHAC1 gene. While the effects of ATF4 on CHAC1 expression have been reported, the current study was the first to demonstrate that XBP1 and ATF6 also upregulated $\mathrm{CHACl}$ expression, to the best of our knowledge.

There are several limitations to the present study. First, only the expression of the key UPR regulators, ATF4, XBP1 and ATF6, were knocked down in PLC cells. Since the endogenous expression levels of these factors are already abundant in PLC cells stimulated with DHA, in the present study, knockdown of their expression was deemed more appropriate. Activation of UPR branches by different stimuli leads to different results in HCC cells, both pro-apoptotic (39) and anti-apoptotic (40) results have been reported before. Simple overexpression of ATF4, XBP1 or ATF6 in HCC cells without DHA may demonstrate their own contribution to the viability of HCC cells. However, such effects may not be related to DHA. Nonetheless, to further elucidate the roles of the activated UPR in DHA-induced ferroptosis, further studies should investigate the effects of the overexpression of these regulators in PLC cells. Second, several binding sites for XBP1 and ATF6 were identified on the $\mathrm{CHAC} 1$ promoter. Although the aim of the present study was to demonstrate how DHA affected the promoter activity of $\mathrm{CHACl}$, the specific locations that these UPR sensors bind to remains to be determined.

In conclusion, the findings of the present study suggested that DHA may effectively induce ferroptosis and activate the UPRs in PLC cells. The knockdown of key regulators of the UPRs suppressed the toxic effects of DHA on PLC cells, which was demonstrated through the downregulation of CHAC1 expression, a key gene responsible for GSH degradation. DHA also promoted the transcription of $\mathrm{CHACl}$. These findings may provide novel insights into the antitumor effects of DHA, suggesting that the DHA-induced UPR promotes cell death in PLC.

\section{Acknowledgements}

Not applicable.

\section{Funding}

No funding was received.

\section{Availability of data and materials}

All data generated and/or analyzed during the present study are included in the published article. 


\section{Authors' contributions}

$\mathrm{ZW}$ and TB designed the study, performed the experiments and analyzed the data. ZW drafted the manuscript. ML, YL and $Z Q$, performed the experiments and assisted with data analysis. LY and BL performed the experiments and revised the manuscript. All authors have read and approved the final manuscript. ZW and TB confirmed the authenticity of the data.

\section{Ethics approval and consent to participate}

All institutional and national guidelines for the care and use of laboratory animals were followed. The present study was approved by the Ethics Committee of Zhengzhou University (Zhengzhou, China).

\section{Patient consent for publication}

Not applicable.

\section{Competing interests}

The authors declare that they have no competing interests.

\section{References}

1. Yang JD, Hainaut P, Gores GJ, Amadou A, Plymoth A and Roberts LR: A global view of hepatocellular carcinoma: Trends, risk, prevention and management. Nat Rev Gastroenterol Hepatol 16: 589-604, 2019.

2. El-Serag HB and Rudolph KL: Hepatocellular carcinoma: Epidemiology and molecularcarcinogenesis. Gastroenterology 132 : 2557-2576, 2007.

3. Tang A, Hallouch O, Chernyak V, Kamaya A and Sirlin CB: Epidemiology of hepatocellular carcinoma: Target population for surveillance and diagnosis. Abdom Radiol (NY) 43: 13-25, 2018

4. El-Serag HB: Epidemiology of viral hepatitis and hepatocellular carcinoma. Gastroenterology 142: 1264-1273 e1261, 2012.

5. Tabrizian P, Jibara G, Shrager B, Schwartz M and Roayaie S: Recurrence of hepatocellular cancer after resection: Patterns, treatments, and prognosis. Ann Surg 261: 947-955, 2015.

6. Shiina S, Sato K, Tateishi R, Shimizu M, Ohama H, Hatanaka T, Takawa M, Nagamatsu H and Imai Y: Percutaneous ablation for hepatocellular carcinoma: Comparison of various ablation techniques and surgery. Can J Gastroenterol Hepatol 2018: 4756147, 2018.

7. Dixon SJ, Lemberg KM, Lamprecht MR, Skouta R, Zaitsev EM, Gleason CE, Patel DN, Bauer AJ, Cantley AM, Yang WS, et al: Ferroptosis: An iron-dependent form of nonapoptotic cell death. Cell 149: 1060-1072, 2012.

8. Hirschhorn T and Stockwell BR: The development of the concept of ferroptosis. Free Radic Biol Med 133: 130-143, 2019.

9. Cao JY and Dixon SJ: Mechanisms of ferroptosis. Cell Mol Life Sci 73: 2195-2209, 2016.

10. Okada H and Mak TW: Pathways of apoptotic and non-apoptotic death in tumour cells. Nat Rev Cancer 4: 592-603, 2004.

11. Hassannia B, Vandenabeele $P$ and Vanden Berghe T: Targeting ferroptosis to iron out cancer. Cancer Cell 35: 830-849, 2019.

12. Stockwell BR, Friedmann Angeli JP, Bayir H, Bush AI, Conrad M, Dixon SJ, Fulda S, Gascón S, Hatzios SK, Kagan VE, et al: Ferroptosis: A regulated cell death nexus linking metabolism, redox biology, and disease. Cell 171: 273-285, 2017.

13. Kagan VE, Mao G, Qu F, Angeli JP, Doll S, Croix CS, Dar HH, Liu B, Tyurin VA, Ritov VB, et al: Oxidized arachidonic and adrenic PEs navigate cells to ferroptosis. Nat Chem Biol 13 81-90, 2017.

14. Ho WE, Peh HY, Chan TK and Wong WS: Artemisinins: Pharmacological actions beyond anti-malarial. Pharmacol Ther 142: 126-139, 2014.
15. Ooko E, Saeed ME, Kadioglu O, Sarvi S, Colak M, Elmasaoudi K, Janah R, Greten HJ and Efferth T: Artemisinin derivatives induce iron-dependent cell death (ferroptosis) in tumor cells. Phytomedicine 22: 1045-1054, 2015.

16. Wang N, Zeng GZ, Yin JL and Bian ZX: Artesunate activates the ATF4-CHOP-CHAC1 pathway and affects ferroptosis in Burkitt's Lymphoma. Biochem Biophys Res Commun 519: 533-539, 2019.

17. Eling N, Reuter L, Hazin J, Hamacher-Brady A and Brady NR: Identification of artesunate as a specific activator of ferroptosis in pancreatic cancer cells. Oncoscience 2: 517-532, 2015.

18. Wang D, Meng G, Zheng M, Zhang Y, Chen A, Wu J and Wei J: The Glutaminase-1 inhibitor 968 enhances dihydroartemisinin-mediated antitumor efficacy in hepatocellular carcinoma cells. PLoS One 11: e0166423, 2016.

19. Zhang CZ, Zhang H, Yun J, Chen GG and Lai PB: Dihydroartemisinin exhibits antitumor activity toward hepatocellular carcinoma in vitro and in vivo. Biochem Pharmacol 83: 1278-1289, 2012

20. Lin R, Zhang Z, Chen L, Zhou Y, Zou P, Feng C, Wang L and Liang G: Dihydroartemisinin (DHA) induces ferroptosis and causes cell cycle arrest in head and neck carcinoma cells. Cancer Lett 381: 165-175, 2016.

21. Du J, Wang T, Li Y, Zhou Y, Wang X, Yu X, Ren X, An Y, Wu Y, Sun W, et al: DHA inhibits proliferation and induces ferroptosis of leukemia cells through autophagy dependent degradation of ferritin. Free Radic Biol Med 131: 356-369, 2019.

22. Chen Y, Mi Y, Zhang X, Ma Q, Song Y, Zhang L, Wang D, Xing J, Hou B, Li H, et al: Dihydroartemisinin-induced unfolded protein response feedback attenuates ferroptosis via PERK/ATF4/HSPA5 pathway in glioma cells. J Exp Clin Cancer Res 38: 402, 2019.

23. Cubillos-Ruiz JR, Bettigole SE and Glimcher LH: Tumorigenic and immunosuppressive effects of endoplasmic reticulum stress in cancer. Cell 168: 692-706, 2017.

24. Wang $M$ and Kaufman RJ: The impact of the endoplasmic reticulum protein-folding environment on cancer development. Nat Rev Cancer 14: 581-597, 2014.

25. Chen D, Fan Z, Rauh M, Buchfelder M, Eyupoglu IY and Savaskan N: ATF4 promotes angiogenesis and neuronal cell death and confers ferroptosis in a xCT-dependent manner. Oncogene 36: 5593-5608, 2017.

26. Fang C, Dai CY, Mei Z, Jiang MJ, Gu DN, Huang Q and Tian L: microRNA-193a stimulates pancreatic cancer cell repopulation and metastasis through modulating TGF-beta2/TGF-betaRIII signalings. J Exp Clin Cancer Res 37: 25, 2018.

27. Tadvalkar G, Pal-Ghosh S, Pajoohesh-Ganji A and Stepp MA: The impact of euthanasia and enucleation on mouse corneal epithelial axon density and nerve terminal morphology. Ocul Surf 18: 821-828, 2020.

28. Luo M, Wu L, Zhang K, Wang $\mathrm{H}$, Zhang T, Gutierrez L, O'Connell D, Zhang P, Li Y, Gao T, et al: miR-137 regulates ferroptosis by targeting glutamine transporter SLC1A5 in melanoma. Cell Death Differ 25: 1457-1472, 2018.

29. Amaral JD, Castro RE, Steer CJ and Rodrigues CM: p53 and the regulation of hepatocyte apoptosis: Implications for disease pathogenesis. Trends Mol Med 15: 531-541, 2009.

30. Banjac A, Perisic T, Sato H, Seiler A, Bannai S, Weiss N, Kölle P, Tschoep K, Issels RD, Daniel PT, et al: The cystine/cysteine cycle: A redox cycle regulating susceptibility versus resistance to cell death. Oncogene 27: 1618-1628, 2008.

31. Jiang L, Kon N, Li T, Wang SJ, Su T, Hibshoosh H, Baer R and $\mathrm{Gu}$ W: Ferroptosis as a p53-mediated activity during tumour suppression. Nature 520: 57-62, 2015.

32. Hou J, Wang D, Zhang R and Wang H: Experimental therapy of hepatoma with artemisinin and its derivatives: In vitro and in vivo activity, chemosensitization, and mechanisms of action. Clin Cancer Res 14: 5519-5530, 2008.

33. Ursini F, Maiorino M, Valente M, Ferri L and Gregolin C: Purification from pig liver of a protein which protects liposomes and biomembranes from peroxidative degradation and exhibits glutathione peroxidase activity on phosphatidylcholine hydroperoxides. Biochim Biophys Acta 710: 197-211, 1982.

34. Dixon SJ, Patel DN, Welsch M, Skouta R, Lee ED, Hayano M, Thomas AG, Gleason CE, Tatonetti NP, Slusher BS and Stockwell BR: Pharmacological inhibition of cystine-glutamate exchange induces endoplasmic reticulum stress and ferroptosis. Elife 3: $\mathrm{e} 02523,2014$. 
35. Walter F, Schmid J, Dussmann H, Concannon CG and Prehn JH: Imaging of single cell responses to ER stress indicates that the relative dynamics of IRE1/XBP1 and PERK/ATF4 signalling rather than a switch between signalling branches determine cell survival. Cell Death Differ 22: 1502-1516, 2015.

36. Szegezdi E, Logue SE, Gorman AM and Samali A: Mediators of endoplasmic reticulum stress-induced apoptosis. EMBO Rep 7: 880-885, 2006

37. BaiT,LiangR,Zhu R, Wang W,ZhouLandSun Y:MicroRNA-214-3p enhances erastin-induced ferroptosis by targeting ATF4 in hepatoma cells. J Cell Physiol 235: 5637-5648, 2020.

38. Kumar A, Tikoo S, Maity S, Sengupta S, Sengupta S, Kaur A and Bachhawat AK: Mammalian proapoptotic factor $\mathrm{ChaC1}$ and its homologues function as gamma-glutamyl cyclotransferases acting specifically on glutathione. EMBO Rep 13: 1095-1101, 2012.
39. Ma MKF, Lau EYT, Leung DHW, Lo J, Ho NPY, Cheng LKW, Ma S, Lin CH, Copland JA, Ding J, et al: Stearoyl-CoA desaturase regulates sorafenib resistance via modulation of ER stress-induced differentiation. J Hepatol 67: 979-990, 2017.

40. Nakagawa H, Umemura A, Taniguchi K, Font-Burgada J, Dhar D, Ogata H, Zhong Z, Valasek MA, Seki E, Hidalgo J, et al: ER stress cooperates with hypernutrition to trigger TNF-dependent spontaneous HCC development. Cancer Cell 26: 331-343, 2014.

(i) (3) This work is licensed under a Creative Commons Attribution-NonCommercial-NoDerivatives 4.0 International (CC BY-NC-ND 4.0) License. 